\title{
Three-wavelength system for practical application in skin oximetry: simultaneous equations with prediction-correction approach
}

\author{
Audrey Huong, Wan Mahani Hafizah Wan Mahmud, Xavier Ngu \\ Faculty of Electrical and Electronic Engineering, Universiti Tun Hussein Onn Malaysia, Malaysia
}

\begin{tabular}{|c|c|}
\hline Article Info & ABSTRACT \\
\hline Article history: & \multirow{12}{*}{$\begin{array}{l}\text { This paper presented the use of a three-wavelength system coupled with } \\
\text { a prediction-correction model for the measurement of a person's tissue } \\
\text { oxygen levels and in the efforts towards the development of a field-portable } \\
\text { system. This study considered light wavelength of market-available emitters } \\
\text { in the range } 500-650 \mathrm{~nm} \text { for its practical implementation. This approach } \\
\text { required the use of light attenuation and hemoglobin absorptivity information } \\
\text { of three different wavelengths in determining tissue oxygen saturation value, } \\
\text { StO2. It was found through the analysis of results using Monte Carlo method } \\
\text { that considerable improvement in the accuracy of the predictions was } \\
\text { obtained using the corrective models }(\rho=0.874) \text {. The low mean prediction } \\
\text { errors of similar magnitude, not exceeding } 4 \% \text {, given by two wavelength } \\
\text { combinations } 538,560,633 \text { nm and } 538,560,650 \text { nm were observed for } \\
\text { signals with signal-noise ratio (SNR) of down to } 30 \mathrm{~dB} \text {. A significant } \\
\text { statistical difference was found between the prediction errors and the } \\
\text { wavelength combination used under this noise condition ( } \rho=0.011 \text { ). } \\
\text { This work concluded that the findings of this study provide insights into } \\
\text { technology implementation of skin oximetry and the possible impacts it } \\
\text { might have in medical arena. }\end{array}$} \\
\hline Received Feb 2, 2020 & \\
\hline Revised Mar 6, 2020 & \\
\hline Accepted Mar 22, 2020 & \\
\hline Keywords: & \\
\hline Corrective model & \\
\hline Simultaneous equations & \\
\hline Skin oximetry & \\
\hline Three wavelengths & \\
\hline Tissue oxygen saturation & \\
\hline & \\
\hline & \\
\hline
\end{tabular}

Copyright () 2020 Institute of Advanced Engineering and Science. All rights reserved.

\section{Corresponding Author:}

Audrey Huong,

Faculty of Electrical and Electronic Engineering,

Universiti Tun Hussein Onn Malaysia,

86400 Batu Pahat, Johor, Malaysia.

Email: audrey@uthm.edu.my

\section{INTRODUCTION}

Oxygen is an important element for life. An interruption in the supply of oxygen to tissue cells especially that in human brain, can have fatal consequences. An adequate supply of tissues with oxygen complemented with active uptake of nutrients, and coupled with a person's positive general physical and environmental conditions would permit favorable cell viability and growth. The importance of the information on tissue oxygen levels in microcirculation systems has been highlighted in many clinical studies, which include its use as a marker for clinical assessment of tissue vascularization in the research of tumor [1] and graft outcome [2], for the evaluation of wound healing [3] and in interventional clinical studies [4].

Various technologies emerged in recent years to determine tissue oxygen consumption in different skin areas of a body (both human and animal); these include the use of pressure oxygen tension technique, opto-acoustic system [5], non-invasive Positron Emission Tomography (PET) [6-7], spectroscopy approach [8-9] and a hybrid of aforementioned approaches [10]. Among these techniques optical spectroscopy is a widely adopted approach that has attracted increasing attention owing to the system's inherent flexibility, cost efficiency and simplicity for use. The next new idea related to this non-invasive system includes its 
utility for real time applications with high portability. This is viable with a system exhibiting fast data acquisition and processing capabilities, and through optimal design of system parameters.

The common strategy in the prediction of tissue oxygen saturation level, $S_{t} O_{2}$, using optical spectroscopy technique involves detection of lights of different wavelength, which have sufficiently propagated in the investigated medium before backscattered from, or transmitted through, the tissue under study. Therefore the appropriate choice of light wavelengths that carry the most significant information is often considered as a key factor in the design of a spectroscopy system. The earliest arterial blood oxygen saturation measurement device involved the use of a heating probe for application on ear [11]. This device allowed measurement of signals of red and green lights for the interpretation of arterial blood oxygenation status. This concept of dual-wavelength spectrometry is adopted in worldwide clinically recognized pulse oximeters for their application on extremities. Due to the nature of clinical trials and ethic populations used in the sampling of calibration data, reliability of pulse oximeters is often a subject for dispute [12-13]. This is owing to its inconsistencies when use on individuals of other ethnic origins or under hypoxic conditions.

Many recent investigations have advanced from pulse oximetry technology. While some attempted to eliminate the use of calibration data in their prediction, others improved the system application and process by adopting non-contact imaging approach to produce two-dimensional information of $S_{t} O_{2}$. The latter is feasible with the use of either a hyperspectral or multispectral imaging system. Earlier studies [14-15] demonstrated the use of lights of three wavelengths carefully chosen using simulation results for oximetry. These authors argued that an important factor affecting accuracy of the prediction is the sufficiency of the employed model in describing light propagation behavior in a scattering-absorbing medium. Such works, however, have not taken into account market availability and other practical aspects of their chosen wavelengths. Others $[8,16-17]$ demonstrated the use of more light wavelength bands in the proposed information processing strategies to increase the prediction accuracy and robustness of the analysis system. This was further supported by previous studies [18-19] revealing a strong light reflectance contrast with the variability in hemoglobin absorptivity in the optical diagnostic window across visible light range of 500 to $650 \mathrm{~nm}$. These proposals have, however, posed a great setback in terms of either placing a high demand on the system's speed performance or increasing system complexity and bulkiness.

The above mentioned approaches, each with their own strengths and weaknesses, have contributed significantly to our understanding of microcirculation systems. These imaging systems are often limited for laboratory use, so there remains a gap for developing a lightweight and portable device for measurement of tissue oxygen level on any parts of a human body. This paper aims to investigate and discuss the feasibility of realizing a field-portable device for calibration-free and non-invasive imaging of tissue oxygenation status using a three-wavelength system with corrective model. In this study, for the first time, the optimum wavelengths for skin oximetry are chosen from those that are readily available in market.

\section{RESEARCH METHOD}

\subsection{Monte carlo simulation of light propagation}

This study begins with simulation of light propagation in a medium that exhibits similar optical properties as human skin. The Monte Carlo (MC) simulation model [20] was used here for the investigation work due to its known blood oxygen saturation value in the medium. The simulation results from this MC model was previously shown to be in good agreement with experimental data of absorbing-scattering phantom media (refer to [21] for the details). In this study, human skin was modeled as a two-layered medium with homogeneously distributed scattering and absorbing centers: the upper layer was an infinite slab of thickness $100 \mu \mathrm{m}$ superimposed on a semi-infinite bottom layer.

While this work assumed the two-layered medium to have the same light scattering properties (i.e. reduced scattering coefficient, $\mu_{s}{ }^{\prime}$ ) shown in Figure 1, the upper layer was a hemoglobin-free medium, which light absorption was entirely by that of melanosomes. The wavelength dependent melanin light absorption, $\mu_{a(m)}$, in units of $\mathrm{mm}^{-1}$ is given by [22]:

$$
\mu_{a(m)}=5 \times 10^{9} \lambda^{-3.33}
$$

where light wavelength, $\lambda$, is in units of $\mathrm{nm}$. The light absorption in this layer is given by the product of $\mu_{a(m)}$ and volume fraction of melanosomes, $f_{v(\text { mel })}$, which was taken here as $2 \%$. This value is in agreement with that of fair skinned individuals [20]. This work considered oxyhemoglobin, $\mathrm{HbO}_{2}$, and deoxyhemoglobin, $H b$, as light absorbing hemoglobin components in the bottom layer of the modeled system following discussions on the negligible light absorption by other chromophores across the considered visible light range in healthy tissue [23-24]. Light absorptivity of this semi-infinite medium, $\mu_{a(b)}$, in $\mathrm{mm}^{-1}$ is linearly related to $\mathrm{S}_{t} \mathrm{O}_{2}$ given by: 


$$
\mu_{a(b)}=\left(\left(\varepsilon_{H b O 2}-\varepsilon_{H b}\right) S_{t} O_{2}+\varepsilon_{H b}\right) T
$$

where the extinction coefficient, $\varepsilon$, to which each hemoglobin component referred is indicated by its subscript. The extinction coefficients of these hemoglobins are shown in Figure 1 while total (whole blood) hemoglobin concentration, $T$, was assumed as $268 \mu \mathrm{molL}^{-1}$. The wavelength dependent melanin light absorption and $S_{t} O_{2}$ dependent $\mu_{a(b)}$ given in (2) were introduced into the upper and bottom layer of the modeled system.

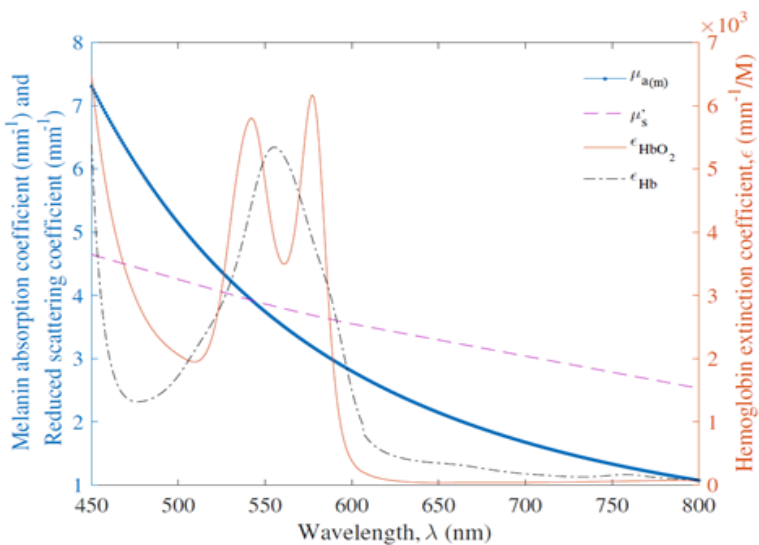

Figure 1. Wavelength dependent optical properties used in simulation model. $\mu_{a(m)}$ : melanin absorption coefficient, $\mu_{s}^{\prime}$ : reduced scattering coefficient, $\mathrm{HbO}_{2}$ : oxyhemoglobin, $\mathrm{Hb}$ : deoxyhemoglobin

The $S_{t} O_{2}$ in (2) is given by the percent ratio of oxy-to total hemoglobin concentration. In this work this value was varied from 30 to $85 \%$ at a step of $5 \%$, producing changes in the optical properties of the lower medium. The choice of this range was based on literature findings [9, 18-19] for the reported most probable tissue oxygen levels determined for different skin regions of humans using multispectral and hyperspectral systems, which were used to capture a series of data in the wavelength of visible range. Since the spatial resolution of these spectroscopy systems is poor, the $S_{t} O_{2}$ is the average of tissue oxygen levels in the imaged microvascular networks.

The light source used in this simulation was an infinitely thin pencil-beam of photons launched at normal incidence. Both the emitter and annular detector shown in Figure 2 were placed in contact with the medium. The propagation behavior of one million photons traveled in this scattering-absorbing medium was traced and the cutoff photon pathlength is given by $1000 \mathrm{~mm}$. The time dependent distribution of photons back-scattered and exited the medium within the numerical aperture of the detector with inner and outer detection radius of $2 \mathrm{~mm}$ and $4 \mathrm{~mm}$, respectively, was recorded. Also shown in this figure is an example of banana-shaped light paths indicating the most probable paths of photons between the source and detector in a turbid medium. Measurement of light attenuation (O.D.) is required in the calculation of $S_{t} O_{2}$, it is given by the logarithm of fraction of photons detected.

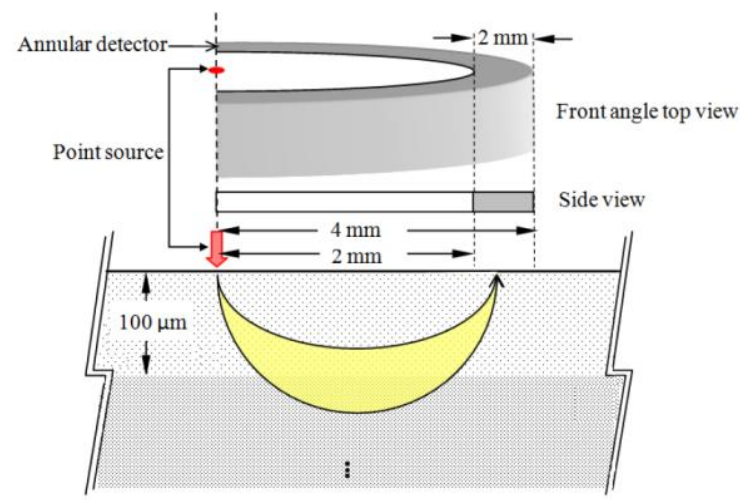

Figure 2. Light transport in a two-layered skin model 
This work considered also statistical fluctuations in the measured signals due to the existence of system noises and measurement uncertainties. For this purpose, normal gaussian white noise (AWGN) was used as the noise source and added in the MC simulated light attenuation spectra to generate noise-embedded signals with SNR of 40, 30 and $20 \mathrm{~dB}$ using MATLAB (version 2016a).

\subsection{Simultaneous equations system}

The prediction of $S_{t} O_{2}$ was via a linear wavelength dependent light attenuation equation given in (3). This equation is the assumption made on the relationship between light attenuation, $A$, and light absorption, $\mu_{a}$, in an absorbing-scattering medium.

$$
A(\lambda)=G \lambda+\mu_{a}(\lambda) d
$$

This model is empirically remodeled from the well known Modified Lambert Beer law (MLBL) by allowing the term $G$, which is often taken as scattering dependent attenuation offset, to vary linearly with the wavelength. This is to approximate the relatively linear changes in light attenuation due to wavelength dependent light scattering and melanin absorptivity shown in Figure 1. The $\mu_{a}$ is given in (2) to give light attenuation equation as a function of $\mathrm{S}_{t} \mathrm{O}_{2}$.

There are three unknowns in (3) (i.e. $G, d$, and $S_{t} O_{2}$ in $\mu_{a}$ ), thus the use of information of three wavelengths (i.e. $\lambda_{1}, \lambda_{2}$ and $\lambda_{3}$ ) in (3) shown by (4)-(6) would sufficiently provide the solution of these unknowns.

$$
\begin{aligned}
& A\left(\lambda_{1}\right)=G \lambda_{1}+\left(\left(\varepsilon_{H b O 2}\left(\lambda_{1}\right)-\varepsilon_{H b}\left(\lambda_{1}\right)\right) S_{t} O_{2}+\varepsilon_{H b}\left(\lambda_{1}\right)\right) d T \\
& A\left(\lambda_{2}\right)=G \lambda_{2}+\left(\left(\varepsilon_{H b O 2}\left(\lambda_{2}\right)-\varepsilon_{H b}\left(\lambda_{2}\right)\right) S_{t} O_{2}+\varepsilon_{H b}\left(\lambda_{2}\right)\right) d T \\
& A\left(\lambda_{3}\right)=G \lambda_{1}+\left(\left(\varepsilon_{H b O 2}\left(\lambda_{3}\right)-\varepsilon_{H b}\left(\lambda_{3}\right)\right) S_{t} O_{2}+\varepsilon_{H b}\left(\lambda_{3}\right)\right) d T
\end{aligned}
$$

Herein, the $S_{t} O_{2}$ is obtained by solving the simultaneous equations shown by (4)-(6) to give

$$
S_{t} O_{2}=\frac{A_{1}\left(\lambda_{2} \varepsilon_{H b 3}-\lambda_{3} \varepsilon_{H b 2}\right)+A_{2}\left(\lambda_{3} \varepsilon_{H b 1}-\lambda_{1} \varepsilon_{H b 3}\right)+A_{3}\left(\lambda_{1} \varepsilon_{H b 2}-\lambda_{2} \varepsilon_{H b 1}\right)}{A_{1}\left(\lambda_{3} \Delta \varepsilon_{2}-\lambda_{2} \Delta \varepsilon_{3}\right)+A_{2}\left(\lambda_{1} \Delta \varepsilon_{3}-\lambda_{3} \Delta \varepsilon_{1}\right)+A_{3}\left(\lambda_{2} \Delta \varepsilon_{1}-\lambda_{1} \Delta \varepsilon_{2}\right)}
$$

where the subscript in symbol $A$ and $\varepsilon$ denotes the wavelength index. The $\Delta \varepsilon_{n}$ is given by subtracting $\varepsilon_{H b}\left(\lambda_{\mathrm{n}}\right)$ from $\varepsilon_{\mathrm{HbO} 2}\left(\lambda_{\mathrm{n}}\right)$ value.

\subsection{Three-wavelength system}

Light attenuation and hemoglobin light absorptivity at three different wavelengths are required in the prediction of $S_{t} O_{2}$ as shown by (7). This study considered only lights of visible wavelengths in the range of 500 to $650 \mathrm{~nm}$; the chosen market available light sources of either coherent or non-coherent type emitting lights within this range are presented in Table 1 . These light wavelength values are separated by at least 10 $\mathrm{nm}$ of wavelength to prevent overlapping of wavelength range due to the broad spectrum of incoherent sources. Meanwhile the coherent sources listed in Table 1 have irradiance value of no more than $1 \mathrm{mWcm}^{-2}$ as in accordance to the maximum exposure limit permitted by laser safety standards. Each diode has physical dimensions not exceeding $10 \mathrm{~mm} \times 10 \mathrm{~mm}$ and current rating of less than $200 \mathrm{~mA}$ for the ease and practical integration into portable devices.

Twenty sets of three wavelengths combined from that listed in Table 1 and arranged in non-specific order were evaluated in terms of accuracy in the predictions. Using the simulation results obtained in section 2.1, the information correspond to each combination of three wavelengths was substituted into (7) to calculate for the required $\mathrm{S}_{t} \mathrm{O}_{2}$ value.

Table 1. Specifications of the chosen market available light sources

\begin{tabular}{cccc}
\hline Num & Part No. (manufacturer) & Type & Center wavelength, $\lambda_{c}(\mathrm{~nm})$ \\
\hline 1 & LED525L (Thorlabs Inc) & non-coherent & 525 \\
2 & LNJ647W8CRA (Panasonic) & non-coherent & 538 \\
3 & LP T655-Q1R2-25 (OSRAM) & non-coherent & 560 \\
4 & LED600L (Thorlabs Inc) & non-coherent & 600 \\
5 & HL63163DG (Thorlabs Inc) & coherent & 633 \\
6 & L650P007 (Thorlabs Inc) & coherent & 650 \\
\hline
\end{tabular}




\section{RESULTS AND ANALYSIS}

The accuracy of the $S_{t} O_{2}$ value determined from (7) using information of each three-wavelength combination was investigated. For ease of viewing, the performance of five combinations giving the least error in their predictions is plotted against the actual $S_{t} O_{2}$ used in simulations in Figure 3 . The prediction error, $\Delta S_{t} O_{2}$, is given by subtracting the estimated value from that used in the simulation. The dotted line, drawn for reference, is the zero level.

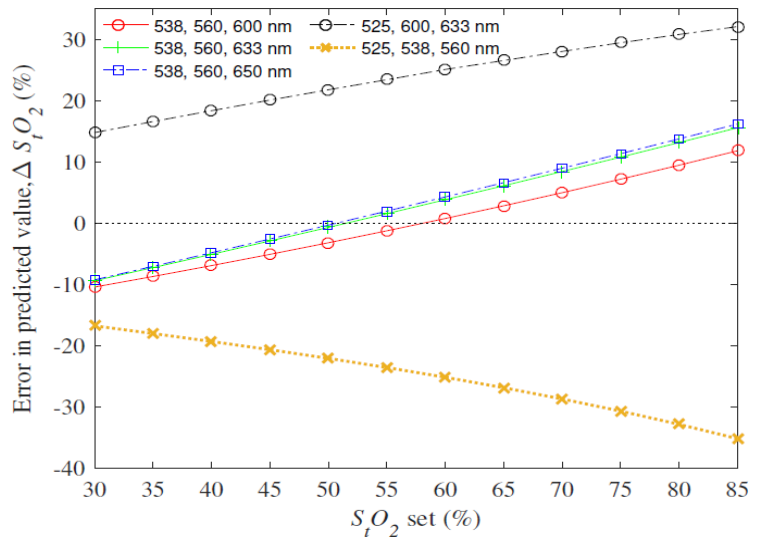

Figure 3. Error in the predicted $\mathrm{S}_{t} \mathrm{O}_{2}$ versus the value set in simulation for the selected three-wavelength combinations

Based on the results in Figure 3 wavelength combinations of 538, 560 and $\{600,633,650\} \mathrm{nm}$, wherein wavelength value inside the curly parentheses indicates the selection of either one, yield similar results and remarkably lower error in their predictions. These combinations of wavelengths produced an average absolute mean (standard deviation, SD) error of $6.78(0.64) \%$. For the ease of analysis, this study focused on the performance of these three combinations for their application in practice. A plot of the predicted values, $S_{\text {est }}$, against the value used in simulations, $S_{\text {act }}$, for these chosen combinations is shown in Figure 4. The relationship between the $S_{\text {est }}$ and $S_{\text {act }}$ is considerably linear for these combinations, thus linear regression model was used to correct for the predicted value. The best fitted linear regression line and the residuals of the fit (represented by symbol $e$ ) for each wavelength combination is also shown in this diagram. These regression equations were used as corrective models to approximate the actual $S_{t} O_{2}$.

The investigation of the performance of this analytical approach for practical use was achieved by considering ten sets of noise-corrupted data of different SNR levels discussed in section 2.1. The mean (SD) of $\Delta S_{t} O_{2}$ given by the corrective models for signals with different SNR value is plotted in Figure 5. The average of these values is calculated and summarized in Table 2. The time taken for each prediction was approximately $1.1 \mathrm{~ms}$.

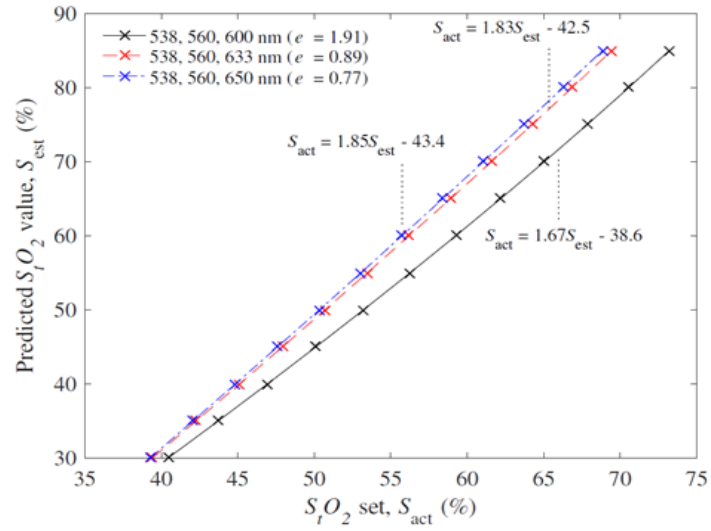

Figure 4. Linear regression model for prediction correction for each wavelength combination. $e$ : Fitting residuals 


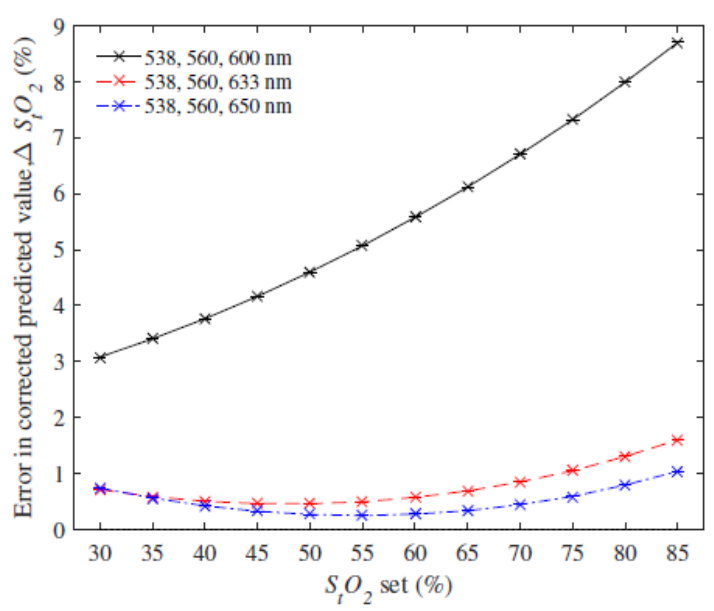

(a)

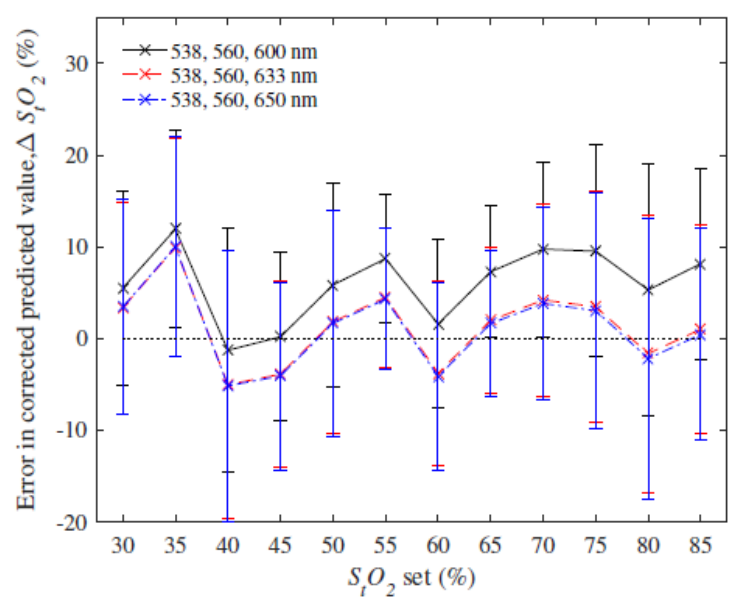

(c)

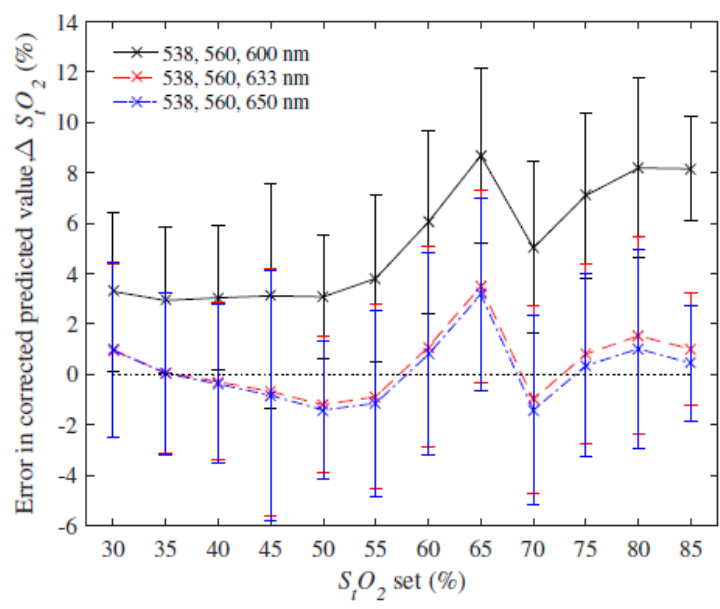

(b)

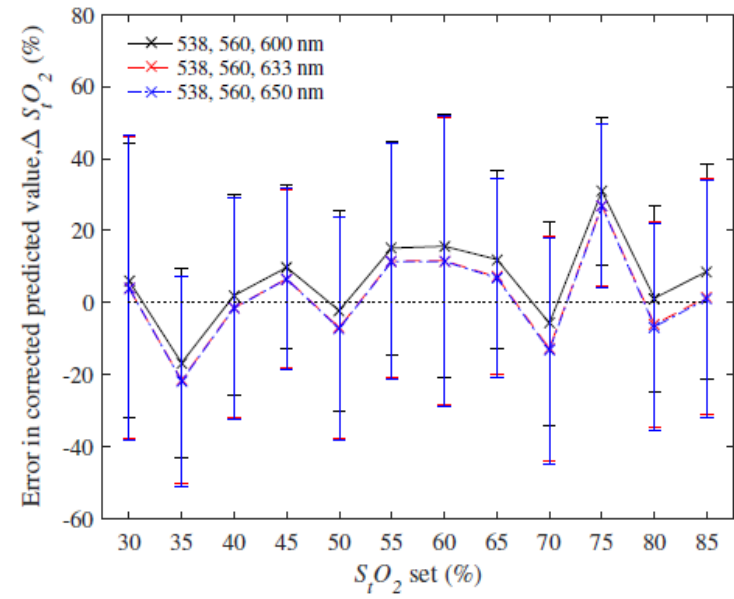

(d)

Figure 5. The calculated error in the prediction corrected values for (a) `noiseless' MC data, and noisecorrupted data with SNR of (b) $40 \mathrm{~dB}$ (c) $30 \mathrm{~dB}$ (d) $20 \mathrm{~dB}$

Table 2. Mean (standard deviation, SD) of absolute error in the prediction corrected values for ten sets of simulation data corrupted with different noise levels

\begin{tabular}{ccccc}
\hline $\begin{array}{c}\text { Wavelength } \\
\text { combination }\end{array}$ & \multirow{2}{*}{ Noiseless } & $40 \mathrm{~dB}$ & $30 \mathrm{~dB}$ & $20 \mathrm{~dB}$ \\
\hline $538,560,600 \mathrm{~nm}$ & $5.54 \%$ & $5.20(3.21) \%$ & $6.24(10.31) \%$ & $10.52(28.23) \%$ \\
$538,560,633 \mathrm{~nm}$ & $0.77 \%$ & $1.08(3.52) \%$ & $3.71(11.31) \%$ & $9.85(30.94) \%$ \\
$538,560,650 \mathrm{~nm}$ & $0.50 \%$ & $0.99(3.56) \%$ & $3.65(11.43) \%$ & $9.89(31.28) \%$ \\
\hline
\end{tabular}

The difference in the results shown in Figure 3 and Figure 5(a) was tested for its statistical significance using a two-tailed paired sample $t$-test (SPSS 22, Inc., Chicago, Illinois) with confidence level of $95 \%$. This statistical test revealed a significance value of $\rho=0.874$. The statistical comparison in the magnitude of errors depending on the wavelength combination used was also performed using ANOVA test for signals with different noise levels. The results revealed a strong correlation with $\rho=0.000$ between the considered wavelength combination and $\Delta \mathrm{S}_{t} \mathrm{O}_{2}$ for both noiseless signals and signals with SNR of $40 \mathrm{~dB}$. While a weak association $(\rho=0.011)$ was found for the relationship between the wavelengths used and the calculated errors for signals with SNR of $30 \mathrm{~dB}$, no significance in this relationship was seen for signals having SNR value of $20 \mathrm{~dB}(\rho=0.561)$. 


\section{DISCUSSION}

Referring to Figure 3, it is interesting to note that, both 538, 560, $633 \mathrm{~nm}$ and 538, 560, $650 \mathrm{~nm}$ combinations produced low error at $S_{t} O_{2}$ of $50 \%$, whereas $538,560,600 \mathrm{~nm}$ showed the same at $S_{t} O_{2}$ of around $60 \%$. An investigation into the light absorptivity of hemoglobins at these wavelengths revealed that light absorption at wavelength $538 \mathrm{~nm}$ and $560 \mathrm{~nm}$ is strongly dominated by $\mathrm{HbO}_{2}$ and $\mathrm{Hb}$, respectively. Meanwhile the light absorptivity by these hemoglobin components is relatively consistent at wavelength 633 $\mathrm{nm}$ and $650 \mathrm{~nm}$. This yields a similar performance in their predictions. While this work observed an underestimation in the value predicted using these combinations in high $S_{t} O_{2}$ regions, the value was appreciably overestimated under low $S_{t} O_{2}$ conditions. The error increased in a considerably linear manner from $S_{t} O_{2}$ of around 50-60\% towards both ends of the range. The main factor contributing to these discrepancies is the assumption made on the linear $\mu$ a and light attenuation relationship. Even though the performance of MLBL was enhanced by incorporating the wavelength varying term (i.e. $G \lambda$ ) in (3), this has only loosely represented the wavelength dependent scattering and melanin absorption effects shown in Figure 1. This error is further accentuated with the poor assumption of the variable $d$, which is taken here as a constant. Photon pathlength in a diffusive medium is a nonlinear function of light absorption and scattering, both of which varied independently with light wavelength.

It is encouraging to observe that the use of corrective models obtained from the results shown in Figure 4 showed promising results by significantly reducing error in the predictions shown by Figure 5(a) ( $\rho$ $=0.874)$. Both 538,560,633 nm and 538,560, $650 \mathrm{~nm}$ combinations produced low errors under moderate noise conditions as compared to $538,560,600 \mathrm{~nm}$ combination. This statistical significance is evidence with the calculated $\rho=0.011$ between the calculated error and wavelengths used for signals with SNR of $30 \mathrm{~dB}$. Based on the comparatively larger norm of residuals of 1.91 for the latter combination shown in Figure 4, these observed errors are likely attributable to the weak representation of the relationship between the $S_{\text {act }}$ and $S_{\text {est }}$ using the deduced model. Nonetheless both the average mean and variation of absolute errors of the former two combinations are found to increase with the signal noise level as shown by Table 2, bringing the values closer to that given by $538,560,600 \mathrm{~nm}$ combination $(\rho=0.561)$. The increase was more substantial in these two combinations, suggesting that the employed corrective models are likely to become unreliable under heavy noise conditions. It must, however, be mentioned that these errors may be minimized using either averaging of more scans or by adopting a pre-processing noise reduction system.

The technology realization of skin oximetry also requires attention on the stability of the emitters and slew rate of optical circuit; this is because changing of illumination wavelength in a sequential order is necessary for measurement of light attenuation needed in the estimation of the required value. The fast response time of detection system is even more crucial for real time applications. Even though this study evaluated the robustness of the proposed analytical strategy by considering signals with different SNR values (i.e. down to $20 \mathrm{~dB}$ ), typical low cost two-dimensional cameras able to produce a signal SNR of approximately $30-40 \mathrm{~dB}$, which is greater than that considered here. This, therefore, relaxes the technical requirement in the light detection system. Other aspects to consider include integrability of light sources and detector in a hollow housing to provide a dark environment for portable measurement of skin oxygenation. This dark chamber is necessary to minimize the effects of stray lights that may affect the measured light reflectance.

Even though the use of this proposed approach is feasible for real time system implementation with average processing time of $1.1 \mathrm{~ms}$, this is at the price of poorer performance stability. By increasing the complexity of the analytic model, for example through the adequate representation of non-linear $\mu_{a}$ and light attenuation relationship [20-25], the accuracy of the prediction could be improved. Such system, however, requires the use of a more advanced processing strategy involving more light wavelength bands, placing a higher demand on the processing power. This alternative also requires a more complex architecture design of a portable device.

\section{CONCLUSION}

This work demonstrated the possibility of using a three-wavelength system for portable and real time measurement of skin oxygen levels. It was found that the optimum three wavelengths comprised of those that have distinctive features in the hemoglobin light absorptivity. Wavelengths combinations 538, 560, $633 \mathrm{~nm}$ and 538,560, $650 \mathrm{~nm}$ were found to produce relatively similar performances and considerably low error in their predictions under moderate noise conditions. The findings of this study can be translated into technology that would exert a positive impact on diagnostic tools and redefine health management systems. 


\section{ACKNOWLEDGEMENTS}

We would like to express our gratitude to Ministry of Education Malaysia (Grant no. FRGS 1581 and PRGS K038) and Universiti Tun Hussein Onn Malaysia (TIER 1 grant no. H223) for in part financial support for the study.

\section{REFERENCES}

[1] M. Welter, T. Fredrich, H. Rinneberg and H. Rieger, "Computational model for tumor oxygenation applied to clinical data on breast tumor hemoglobin concentrations suggests vascular dilatation and compression," PLOS ONE, vol. 11, pp. 1-42, 2016.

[2] P.K. Upputuri, K. Sivasubramanian, S.K. Chong and M. Pramanik, "Recent developments in vascular imaging techniques in tissue engineering and regenerative medicine," BioMed Research International, vol. 2015 pp. 1-10, 2015.

[3] C.K. Sen, S. Ghatak, S.C. Gnyawali, S. Roy and G.M. Gordillo, "Cutaneous imaging technologies in acute burn and chronic wound care," Plast Reconstr Surg., vol.138, pp. 1-20, 2016.

[4] L. Meng, F. Settecase, J. Xiao, Z. Yu, A.M. Flexman and R.T. Higashida, "Initial clinical experience with nearinfrared spectroscopy in assessing cerebral tissue oxygen saturation in cerebral vasospasm before and after intraarterial verapamil injection," AIP: Review of Scientific Instruments, vol.13, pp. 1-12, 1942.

[5] I.Y. Petrov, R.A. Fonseca, C.J. Richardson, Y. Petrov, D.S. Prough, A. Petrov, K.E. Wynne, S. Westermann and R.O. Esenaliev, "Monitoring cerebral venous blood oxygenation in neonates with a medical-grade optoacoustic system," in Proc. SPIE 9323: Photons Plus Ultrasound: Imaging and Sensing, vol.9323, pp. 1-5, 2015.

[6] S.G. Demos, A.J. Vogel and A.H. Gandjbakhche, "Advances in optical spectroscopy and imaging of breast lesions," J Mammary Gland Biol Neoplasia, vol.11, pp.165-181, 2006.

[7] H. Ito, I. Kanno, H. Iida, J. Hatazawa, E. Shimosegawa, H. Tamura and T. Okudera, "Arterial fraction of cerebral blood volume in humans measured by positron emission tomography," Ann Nucl Med, vol. 15, pp. 111-6, 2001.

[8] C. Hsu, S. Tzeng, C. Yang, J.Y. Lee, L.L. Huang, W. Chen, M. Hughes, Y. Chen, Y. Liao and S. Tseng, "Noninvasive evaluation of therapeutic response in keloid scar using diffuse reflectance spectroscopy," Biomedical Optics Express, vol. 6, pp. 390-404, 2015.

[9] A. Huong, S. Philimon and X. Ngu, "Multispectral imaging of acute wound tissue oxygenation," Journal of Innovative Optical Health Sciences, vol.10, pp.17500041-8, 2017.

[10] A. Becker, M. Masthoff, J. Claussen, S.J. Ford, W. Roll, M. Burg, P.J. Barth, W. Heindel, M. Schafers, M. Eisenblatter and M. Wildgruber, "Multispectral optoacoustic tomography of the human breast: characterisation of healthy tissue and malignant lesions using a hybrid ultrasound-optoacoustic approach," Eur Radiol. vol. 28, no. 2, pp. 602 -609, 2018.

[11] G.A. Milikan, "The Oximeter, an instrument for measuring continuously the oxygen saturation of arterial blood in man," Review of Scientific Instruments, vol. 13, pp. 1-7, 1942.

[12] M. Nitzan, S. Noach, E. Tobal, Y. Adar, Y. Miller, E. Shalom and S. Engelberg, "Calibration-Free Pulse Oximetry based on two wavelengths in the Infrared - A preliminary study," Sensors, vol. 14, no. 4, pp. 7420-7434, 2014.

[13] G.D. Perkins, D.F. McAuley, S. Giles, H. Routledge and F. Gao, “ Do changes in pulse oximeter oxygen saturation predict equivalent changes in arterial oxygen saturation?," Critical Care, vol.7, pp. 67-71, 2003.

[14] M.H. Smith, "Optimum wavelength combinations for retinal vessel oximetry," Applied Optics, vol. 38, pp. 258-267, 1999.

[15] A. Huong, I.M. Stockford, J.A. Crowe and S.P. Morgan, "Investigation of optimum wavelengths for oximetry," in Proceedings of SPIE: OSA Biomedical Optics, vol. 7368, pp. 1-7, 2009.

[16] N. Rajaram, T. Nguyen and J.W. Tunnell, "Lookup table based inverse model for determining optical properties of turbid media," Journal of Biomedical Optics, vol. 13, pp. 0505011-3, 2008.

[17] X. Zhong, X. Wen and D. Zhu, "Lookup-table-based inverse model for human skin reflectance spectroscopy: twolayered Monte Carlo simulations and experiments," Optics Express, vol. 22, pp.1852-1864, 2014.

[18] T. Chen, P. Yuen, M. Richardson, Z. She and G. Liu, "Wavelength and model selection for hyperspectral imaging of tissue oxygen saturation,” The Imaging Science Journal, vol. 63, pp. 1-7, 2015.

[19] K.A. Firn and B. Khoobehi, "Noninvasive Multispectral snapshot imaging system to measure and map the distribution of human retinal vessel and tissue hemoglobin oxygen saturation," International Journal of Ophthalmic Research, vol. 1, pp. 48-58, 2015.

[20] A. Huong and X. Ngu. "The application of extended modified Lambert Beer model for measurement of blood carboxyhemoglobin and oxyhemoglobin saturation," Journal of Innovative Optical Health Sciences, vol.7, pp. 145002641-7, 2014.

[21] A. Huong, Spectroscopic analysis of scattering media via different quantification techniques: University of Nottingham, 2012.

[22] I.V. Meglinski and S.J. Matcher, "Quantitative assessment of skin layers absorption and skin reflectance spectra simulation in the visible and near-infrared spectral regions," Physiol. Measurement, vol. 23, no. 4, pp. 741- 753, 2002.

[23] L. Meng, W.W. Mantulin, B.S. Alexander, A.E. Cerussi, B.J. Tromberg, Z. Yu, K. Laning, Z.N. Kain, M. Cannesson and A.W. Gelb, "Head-up tilt and hyperventilation produce similar changes in cerebral oxygenation and blood volume: an observational comparison study using frequency-domain near infrared spectroscopy," Can J Anesth., vol. 59, pp. 357-365, 2012.

[24] D. Yim, G.V.G. Baranoski, B.W. Kimmel, T.F. Chen and E. Miranda, "A cell-based light interaction model for human blood," Eurographics, vol. 31, pp. 845-854, 2012.

[25] V.M. Niemeijer, R.F. Spee, J.P. Jansen, A.B.C. Buskermolen, T.V. Dijk, P.F.F. Wijn and H.M.C. Kemps, "Test retest reliability of skeletal muscle oxygenation measurements during submaximal cycling exercise in patients with chronic heart failure," Clin Physiol Funct Imaging, vol. 37, pp. 68-78, 2017. 


\section{BIOGRAPHIES OF AUTHORS}
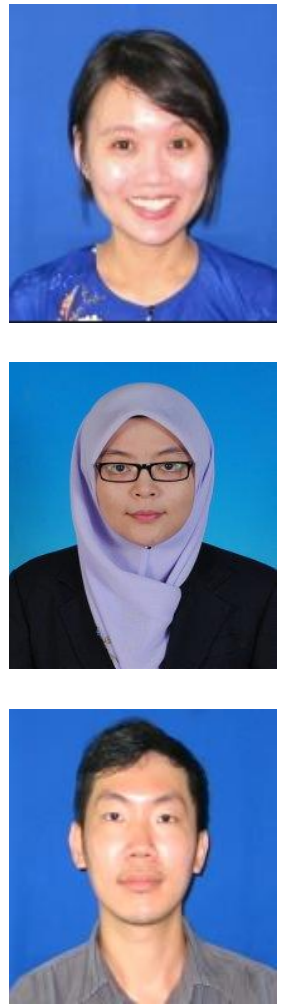

Audrey Huong is a senior researcher and Associate Professor at Universiti Tun Hussein Onn Malaysia. Her research interests include noninvasive physiology monitoring of human health, technology development and artificial intelligence assisted clinical diagnosis.

Wan Mahani Hafizah was born in Kuala Lumpur, Malaysia in 1987. She received her B.E. degree in biomedical engineering from Universiti Teknologi Malaysia, Malaysia, in 2009, and later her $\mathrm{PhD}$ degree in biomedical engineering from the same university in 2013. In 2014, she started joining the Department of Electronic Engineering, Universiti Tun Hussein Onn Malaysia, as a lecturer. Her current research interests include ultrasound imaging, medical image processing, and computer aided system, as well as telemedicine and rehabilitation.

Xavier Ngu is a senior lecturer at Universiti Tun Hussein Onn Malaysia. His research interests include electrostatic discharge protection, electromagnetic compatibility and biomedical imaging. 